\title{
Stability of the Translation Equation in Rings of Formal Power Series and Partial Extensibility of One-Parameter Groups of Truncated Formal Power Series
}

\author{
By \\ Wojciech Jabłoński and Ludwig Reich \\ (Vorgelegt in der Sitzung der math.-nat. Klasse am 12. Oktober 2006 \\ durch das w. M. Ludwig Reich)
}

\begin{abstract}
We prove in this paper that stability of the translation equation in the ring of formal power series $\mathbb{K} \llbracket X]$ (more precisely, in the group of invertible formal series over $\mathbb{K}$ ) is equivalent to some kind of extensibility of one-parameter groups of truncated formal power series. From this we deduce the stability of the translation equation in $\mathbb{K} \llbracket X \rrbracket$.

AMS Subject Classification: 39B82, 39B12, 13F25.

Key words and phrases: Formal power series, translation equation, stability.

\section{Introduction}

Following the famous problem of the stability of the equation of homomorphism posed by S. ULAM (cf. [8]) many authors have studied such problems for various functional equations (see [1] and [2]). We are going to consider here the problem of stability for the translation equation in the ring of formal power series in one indeterminate. Surprisingly, the considered problem is close to the problem of extensibility of one-parameter groups of truncated formal power series.
\end{abstract}


In fact, we will show that the stability of the translation equation in the ring of formal power series is equivalent to the possibility of extension of some restriction of a given truncated formal power series (for the concepts of truncation and extension we refer the reader to $[5])$.

Our main results about the stability of the translation equation (1) in a ring $\mathbb{K} \llbracket X \rrbracket$ of formal power series with $\mathbb{K} \in\{\mathbb{R}, \mathbb{C}\}$ are Theorems 2 and 3 stating Hyers-Ulam stability of the translation equation under a rather mild condition on the group $G$ of the "time parameter" $t$. Let us also mention that the constants in these stability results are best possible.

In this introduction we recall basic notions about formal series and their truncations. Let $\mathbb{N}$ stand for the set of all positive integers and let $\mathbb{K}$ be the field of real or complex numbers. For $k, l \in \mathbb{N}$, by $|k, l|$ we denote the set of all integers $n$ such that $k \leq n \leq l$. Similarly, by $|k, \infty|$ we mean the set of all integers $n$ with $n \geq k$. Throughout this paper we will assume that $|\infty, \infty|=\emptyset, \sum_{t \in \emptyset} a_{t}=0$ and $\prod_{t \in \emptyset} a_{t}=1$.

By $\mathbb{\llbracket} \llbracket X \rrbracket$ we denote the ring of all power series $\sum_{i=0}^{\infty} c_{i} X^{i}$ with coefficients in $\mathbb{K}$. For any formal power series $p(X)=\sum_{i=0}^{\infty} c_{i} X^{i}$ we define

$$
\operatorname{ord} p(X):=\min \left\{i \in \mathbb{N} \cup\{0\}: c_{i} \neq 0\right\}
$$

assuming additionally that $\min \emptyset=\infty$.

The function $d: \mathbb{K} \llbracket X \rrbracket \times \mathbb{K} \llbracket X \rrbracket \rightarrow[0, \infty)$,

$$
d\left(p_{1}(X), p_{2}(X)\right)=\left(1+\operatorname{ord}\left(p_{1}(X)-p_{2}(X)\right)\right)^{-1},
$$

properly defines a metric and $(\mathbb{K} \llbracket X \rrbracket, d)$ is a complete metric space. Moreover, the inequality $d\left(p_{1}(X), p_{2}(X)\right)<\varepsilon$ is equivalent with $\operatorname{ord}\left(p_{1}(X)-p_{2}(X)\right)>(1 / \varepsilon)-1$.

A formal power series $p(X) \in \mathbb{K} \llbracket X \rrbracket$ can be substituted into the series $q(X)=\sum_{i=0}^{\infty} c_{i} X^{i}$ provided ord $p(X) \geq 1$ (only in this case we can properly compute the power series $\left.(q \circ p)(X):=\sum_{i=0}^{\infty} c_{i}(p(X))^{i}\right)$. Denote $\Gamma(\mathbb{K}):=\{p(X) \in \mathbb{K} \llbracket X \rrbracket$ : ord $p(X)=1\}$. Then $\Gamma(\mathbb{K})$ with the substitution as a binary operation is a group.

Finally we will need the notion of congruence modulo $X^{s+1}$ and the notion of truncated formal power series. It is easy to see that for $s \in \mathbb{N}$ the set

$$
I_{s}=\{p(X) \in \mathbb{K} \llbracket X \rrbracket: \text { ord } p(X) \geq s+1\}=X^{s+1} \mathbb{K} \llbracket X \rrbracket
$$


forms an ideal in the ring $\mathbb{K} \llbracket\left[X \rrbracket\right.$. We define a congruence modulo $X^{s+1}$ in the following way. We say that for $p_{1}(X), p_{2}(X) \in \mathbb{K} \llbracket X \rrbracket$ we have

$$
p_{1}(X) \equiv p_{2}(X) \bmod X^{s+1} \Longleftrightarrow p_{1}(X)-p_{2}(X) \in I_{s} .
$$

This clearly means that $X^{s+1}$ is a divisor of $\left(p_{1}-p_{2}\right)(X):=$ $p_{1}(X)-p_{2}(X)$. We consider the quotient ring $\mathbb{K} \llbracket X \rrbracket / I_{s}$ of all cosets $[p(X)]_{s}=p(X)+I_{s}$. With every coset $p(X)+I_{s}$, where $p(X)=$ $\sum_{i=0}^{\infty} c_{i} X^{i} \in \mathbb{K} \llbracket X \rrbracket$, we will associate an s-truncation of a formal power series $p(X)$ defined by

$$
p^{[s]}(X):=\sum_{i=0}^{s} c_{i} X^{i} \in \mathbb{K} \llbracket X \rrbracket_{s} \subset \mathbb{K} \llbracket X \rrbracket .
$$

In the set $\mathbb{K} \llbracket X \rrbracket_{s}$ we introduce, in a natural way, an addition, a multiplication and a substitution as follows: For $p(X), q(X) \in \mathbb{K} \llbracket X \rrbracket_{s}$ let

$$
\begin{gathered}
(p+q)(X)=(p+q)^{[s]}(X), \\
(p q)(X):=(p q)^{[s]}(X),
\end{gathered}
$$

and, in the case when ord $q(X) \geq 1$,

$$
(p \circ q)(X):=(p \circ q)^{[s]}(X) .
$$

Then $\left(\mathbb{K} \llbracket X \rrbracket_{s},+, \cdot\right)$ is a ring which is isomorphic with $\mathbb{K} \llbracket X \rrbracket / I_{s}$. Moreover, the set $\Gamma(\mathbb{K})_{s}:=\left\{p(X) \in \mathbb{K} \llbracket X \rrbracket_{s}\right.$ : ord $\left.p(X)=1\right\}$ is a group under substitution.

Definition 1. By a one-parameter group of formal power series we mean a homomorphism $\Phi: G \rightarrow \Gamma(\mathbb{K})$ from a group $(G,+)$ into $(\Gamma(\mathbb{K}), \circ)$.

For a one-parameter group of formal power series $\Phi: G \rightarrow \Gamma(\mathbb{K})$ let us denote $F_{t}(X)=F(t, X)=\Phi(t)(X)=\sum_{i=1}^{\infty} c_{i}(t) X^{i}$, where $c_{1}: G \rightarrow \mathbb{K} \backslash\{0\}, c_{i}: G \rightarrow \mathbb{K}$ for $i \geq 2$. Then the family $(F(t, X))_{t \in G}$ satisfies the well-known translation equation in the ring of formal power series, i.e.

$$
F\left(t_{1}+t_{2}, X\right)=F\left(t_{1}, F\left(t_{2}, X\right)\right) \quad \text { for } \quad t_{1}, t_{2} \in G,
$$

or, briefly,

$$
F_{t_{1}+t_{2}}=F_{t_{1}} \circ F_{t_{2}} \quad \text { for } \quad t_{1}, t_{2} \in G .
$$

Analogously, we can define a one-parameter group of $s$-truncated formal power series as a homomorphism $\Phi_{s}: G \rightarrow \Gamma(\mathbb{K})_{s}$. If we denote $F_{t}^{[s]}(X)=F^{[s]}(t, X)=\Phi_{s}(t)(X)=\sum_{i=1}^{s} c_{i}(t) X^{i}$, where $c_{1}: G \rightarrow \mathbb{K} \backslash\{0\}$, 
$c_{i}: G \rightarrow \mathbb{K}$ for $2 \leq i \leq s$, then the family $\left(F^{[s]}(t, X)\right)_{t \in G}$ satisfies also the translation equation

$F^{[s]}\left(t_{1}+t_{2}, X\right)=F^{[s]}\left(t_{1}, F^{[s]}\left(t_{2}, X\right)\right) \bmod X^{s+1} \quad$ for $\quad t_{1}, t_{2} \in G$,

in the ring of $s$-truncated formal power series.

Definition 7 contains the notion of stability we are investigating in this paper. It is, in fact, the notion of stability which is used in the theory of the classical Cauchy equation, since by the definition of the metric $d$ Definition 7 can easily be reformulated in terms of this metric. However, for considerations involving formal series the concept of order seems to be more convenient than the related metric $d$.

\section{Stability and Extensibility}

We will show here that the Hyers-Ulam stability of the translation equation (1) in formal power series is equivalent with some special kind of extensibility of one-parameter groups of truncated formal power series.

Let us begin with the problem of extensibility of one-parameter groups of $s$-truncated formal power series (cf. [7]).

Definition 2. Let $(G,+)$ be a group. A one-parameter group $\left(F_{t}^{[s]}(X)\right)_{t \in G}$ of $s$-truncated formal power series is called extensible provided there exists a one-parameter group of $(s+1)$-truncated formal power series $\left(\bar{F}_{t}^{[s+1]}(X)\right)_{t \in G}$ such that

$$
\left(\bar{F}_{t}^{[s+1]}\right)^{[s]}(X)=F_{t}^{[s]}(X) \quad \text { for every } t \in G .
$$

In other words, we ask whether for a one-parameter group of $s$ truncated formal power series $F^{[s]}(t, X)=\sum_{i=1}^{s} c_{i}(t) X^{i}$ there exists a function $c_{s+1}: G \rightarrow \mathbb{K}$ such that $\bar{F}^{[s+1]}(t, X)=\sum_{i=1}^{s+1} c_{i}(t) X^{i}$ is a oneparameter group of $(s+1)$-truncated formal power series.

The partial solution of this problem one can find in [4, 5, 6]. Here we will need another kind of extensibility. Namely

Definition 3. Let $r \in(0,1]$ and let $(G,+)$ be a group. A one-parameter group $F^{[s]}(t, X)=\sum_{i=1}^{s} c_{i}(t) X^{i}, t \in G$ of $s$-truncated formal power series is called $r$-partially $\infty$-extensible if there exists a one-parameter group of formal power series $\bar{F}(t, X)=\sum_{i=1}^{\infty} \bar{c}_{i}(t) X^{i}, t \in G$ such that

$$
(\bar{F})^{[r s]}(t, X)=\left(F^{[s]}\right)^{[r s]}(t, X) \quad \text { for } \quad t \in G
$$

or, equivalently, $c_{i}=\bar{c}_{i}$ for $1 \leq i \leq[r s]$. 
Definition 4. Let $r \in(0,1]$ and let $(G,+)$ be a group. The translation equation (1) in a ring of formal power series is called $r$-stable if for every positive integer $M$ and for every family $F(t, X)=\sum_{i=1}^{\infty} c_{i}(t) X^{i}$, $t \in G$ of formal power series such that

$$
\operatorname{ord}\left(F\left(t_{1}+t_{2}, X\right)-F\left(t_{1}, F\left(t_{2}, X\right)\right)\right)>M \quad \text { for every } t_{1}, t_{2} \in G,
$$

there is a one-parameter group of formal power series $\bar{F}(t, X)=$ $\sum_{i=1}^{\infty} \bar{c}_{i}(t) X^{i}$ such that

$$
\operatorname{ord}(F(t, X)-\bar{F}(t, X))>r M \quad \text { for } \quad t \in G
$$

or, equivalently, $c_{i}=\bar{c}_{i}$ for $1 \leq i \leq[r M]$.

We prove now that $r$-partial $\infty$-extensibility of one-parameter groups of truncated formal power series is equivalent to Hyers-Ulam $r$-stability of the translation equation (1) in rings of formal power series.

Proposition 1. Fix $r \in(0,1]$. The translation equation (1) in the ring of formal power series is $r$-stable if and only if each one-parameter group of truncated formal power series is r-partially $\infty$-extensible.

Proof. Assume that the translation equation in $\mathbb{K} \llbracket X \rrbracket$ is $r$-stable. Fix $s \in \mathbb{N}$ arbitrarily and let $F^{[s]}(t, X)=\sum_{i=1}^{s} c_{i}(t) X^{i}$ be a one-parameter group of $s$-truncated formal power series. Put $F(t, X)=\sum_{i=1}^{\infty} c_{i}(t) X^{i}$, where $c_{i}: G \rightarrow \mathbb{K}$ for $i>s$ are arbitrary. Then

$$
\operatorname{ord}\left(F\left(t_{1}+t_{2}, X\right)-F\left(t_{1}, F\left(t_{2}, X\right)\right)\right)>s \quad \text { for } \quad t_{1}, t_{2} \in G \text {. }
$$

Since the translation equation is $r$-stable, we find a one-parameter group of formal power series $\bar{F}(t, X)=\sum_{i=1}^{\infty} \bar{c}_{i}(t) X^{i}$ such that

$$
\operatorname{ord}(F(t, X)-\bar{F}(t, X))>r s \quad \text { for } \quad t \in G \text {. }
$$

This means that $c_{i}=\bar{c}_{i}$ for $1 \leq i \leq[r s]$, so every one-parameter group of truncated formal power series is $r$-partially $\infty$-extensible.

Now, assume that each one-parameter group of truncated formal power series is $r$-partially $\infty$-extensible. Fix $M \in \mathbb{N}$ and let a family $F(t, X)=\sum_{i=1}^{\infty} c_{i}(t) X^{i}, t \in G$ be such that

$$
\operatorname{ord}\left(F\left(t_{1}+t_{2}, X\right)-F\left(t_{1}, F\left(t_{2}, X\right)\right)\right)>M \quad \text { for } \quad t_{1}, t_{2} \in G \text {. }
$$

Then $F^{[M]}(t, X)=\sum_{i=1}^{M} c_{i}(t) X^{i}$ is a one-parameter group of $M$-truncated formal power series. On account of our assumption there exists a one-parameter group $\bar{F}(t, X)=\sum_{i=1}^{\infty} \bar{c}_{i}(t) X^{i}$ such that $c_{i}=\bar{c}_{i}$ for $1 \leq i \leq[r M]$. Thus

$$
\operatorname{ord}(F(t, X)-\bar{F}(t, X))>r M \quad \text { for } \quad t \in G .
$$

This finishes the proof. 
Thus the problem of stability of the translation equation (1) can be reduced to discussion of extensibility of one-parameter groups of truncated formal power series. We will need a certain form of the general solution of the translation equations (1)-(2) in $\mathbb{R} \llbracket X \rrbracket$. We will assume here that $(G,+)$ is an abelian group such that for every generalized exponential function $f: G \rightarrow \mathbb{K} \backslash\{0\}$ we have either $f=1$ or $f$ takes infinitely many values. This is equivalent to the following assumption

(A) If $\mathbb{K}=\mathbb{R}$ then $G$ has no subgroup of index 2 and if $\mathbb{K}=\mathbb{C}$ then $G$ has no subgroup with a finite index distinct form $G$.

Indeed, for a generalized exponential function $f: G \rightarrow \mathbb{K} \backslash\{0\}, f \neq 1$ taking finitely many values we have $\operatorname{im} f \cong G / \operatorname{ker} f$, which jointly with the form of finite subgroups of $(\mathbb{K} \backslash\{0\}, \cdot)$ gives us the assumption (A). Conversely, if we assume that (A) does not hold, then one can construct a generalized exponential function $f: G \rightarrow \mathbb{K} \backslash\{0\}, f \neq 1$ which takes finitely many values.

We have

Theorem 1 (cf. [4, 5, 6]). Let $s$ be a positive integer or $s=\infty$. Assume that $(G,+)$ is an abelian group satisfying assumption (A). There exist sequences of polynomials $\left(L_{n}^{p+2}\right)_{n \geq p+2}$ and $\left(P_{n}\right)_{n \geq 2}$ such that $F^{[s]}(t, X)=\sum_{i=1}^{s} c_{i}(t) X^{i}, \quad c_{1}: G \rightarrow \mathbb{K} \backslash\{0\}, \quad c_{n}: G \rightarrow \mathbb{K}$ for $n \in|2, s|$ is a solution of the translation equation ((1) if $s=\infty$ and (2) otherwise) if and only if one of three possibilities holds:

(a) $c_{1}=1$ and $c_{n}=0$ for every $n \in|2, s|$,

(b) $c_{1}=1, c_{n}=0$ for $n \in|2, p+1|, c_{p+2}$ is a nonzero additive function and

$$
\begin{aligned}
c_{n}(t)= & h_{n} c_{p+2}(t)+L_{n}\left(c_{p+2}(t), 1, h_{p+3}, \ldots, h_{n-p-1}\right), \\
& n \in|p+3, s-p-1|, \\
c_{n}(t)= & a_{n}(t)+L_{n}\left(c_{p+2}(t), 1, h_{p+3}, \ldots, h_{n-p-1}\right), \quad n \in|s-p, s|,
\end{aligned}
$$

where $\left(h_{n}\right)_{n \in|p+3, s-p-1|}, h_{n} \in \mathbb{K}$ for $n \in|p+3, s-p-1|$, is an arbitrary sequence of constants and $a_{n}: G \rightarrow \mathbb{K}$ for $n \in|s-p, s|$ are arbitrary additive functions,

(c) $c_{1} \neq 1$ is a generalized exponential function and $c_{n}(t)=c_{1}(t)\left(\lambda_{n}\left(c_{1}(t)^{n-1}-1\right)+P_{n}\left(c_{1}(t) ; \lambda_{2}, \ldots, \lambda_{n}\right)\right), \quad n \in|2, s|$, where $\left(\lambda_{n}\right)_{n \geq 2}, \lambda_{n} \in \mathbb{K}$ for $n \geq 2$, is an arbitrary sequence of constants.

Now we will consider the problem of extensibility of one-parameter groups of $s$-truncated formal power series. In fact, we will discuss a more complicated version of extensibility. 
Definition 5 (cf. [7]). Fix $l \in \mathbb{N}$. A one-parameter group $\left(F_{t}^{[s]}(X)\right)_{t \in G}$ of $s$-truncated formal power series is called l-extensible if there exists a one-parameter group of $(s+l)$-truncated formal power series $\left(\bar{F}_{t}^{[s+l]}(X)\right)_{t \in G}$ such that

$$
\left(\bar{F}_{t}^{[s+l]}\right)^{[s]}(X)=F_{t}^{[s]}(X) \quad \text { for every } \quad t \in G .
$$

Thus we ask whether for a one-parameter group of $s$-truncated formal power series $F^{[s]}(t, X)=\sum_{i=1}^{s} c_{i}(t) X^{i}$ there exist functions $c_{s+1}, \ldots$, $c_{s+l}: G \rightarrow \mathbb{K}$ such that $\bar{F}^{[s+l]}(t, X)=\sum_{i=1}^{s+l} c_{i}(t) X^{i}$ is a one-parameter group of $(s+l)$-truncated formal power series.

Definition 6 (cf. [7]). A one-parameter group $\left(F_{t}^{[s]}(X)\right)_{t \in G}$ of $s$-truncated formal power series is called $\infty$-extensible if there exists a one-parameter group of formal power series $\left(\bar{F}_{t}(X)\right)_{t \in G}$ such that

$$
\left(\bar{F}_{t}\right)^{[s]}(X)=F_{t}^{[s]}(X) \quad \text { for every } \quad t \in G .
$$

This means that we ask whether for a one-parameter group of $s$-truncated formal power series $F^{[s]}(t, X)=\sum_{i=1}^{s} c_{i}(t) X^{i}$ we can find functions $c_{s+1}, c_{s+2}, \ldots: G \rightarrow \mathbb{K}$ such that $\bar{F}(t, X)=\sum_{i=1}^{\infty} c_{i}(t) X^{i}$ is a one-parameter group of formal power series.

Now we have all tools to discuss the extension problem for oneparameter groups of truncated formal power series. We will generally assume that $(G,+)$ is an abelian group satisfying (A). By $\mathcal{A}(G, \mathbb{K})$ we denote the vector space over $\mathbb{K}$ of all additive functions $a: G \rightarrow \mathbb{K}$.

We begin with the discussion of the subcase when $\operatorname{dim}_{\mathbb{K}} \mathcal{A}(G, \mathbb{K}) \leq 1$. From Theorem 1 one can easily derive

Corollary 1. Assume that $(G,+)$ is an abelian group satisfying (A) and such that $\operatorname{dim}_{\mathbb{K}} \mathcal{A}(G, \mathbb{K}) \leq 1$. If $F^{[s]}(t, X)=\sum_{i=1}^{s} c_{i}(t) X^{i}$ for $t \in G$, where $c_{1}: G \rightarrow \mathbb{K} \backslash\{0\}, c_{i}: G \rightarrow \mathbb{K}$ for $2 \leq i \leq s$, is a one-parameter group of s-truncated formal power series (this means that $F^{[s]}(t, X)$ is of the form given in Theorem 1), then $\left(F^{[s]}(t, X)\right)_{t \in G}$ is $l$ - and $\infty$-extensible for any $l \in \mathbb{N}$.

If we do not assume that $\operatorname{dim}_{\mathbb{K}} \mathcal{A}(G, \mathbb{K}) \leq 1$ then we have

Corollary 2. Assume that $(G,+)$ is an abelian group satisfying assumption (A). Let $F^{[s]}(t, X)=\sum_{i=1}^{s} c_{i}(t) X^{i}$ for $t \in G$, where $c_{1}: G \rightarrow \mathbb{K} \backslash\{0\}$, $c_{i}: G \rightarrow \mathbb{K}$ for $2 \leq i \leq s$, be a one-parameter group of s-truncated 
formal power series (this means that $F^{[s]}(t, X)$ is of the form given in Theorem 1).

(1) If $c_{1} \neq 1$, then $\left(F^{[s]}(t, X)\right)_{t \in G}$ is $l$ - and $\infty$-extensible.

(2) If $c_{1}=1$, then $\left(F^{[s]}(t, X)\right)_{t \in G}$ is l-extensible if and only if there are constants $d_{i} \in \mathbb{K}$ for $s-p \leq i \leq \min (s-p+l-1, s)$ such that $a_{i}=d_{i} c_{p+2}$ for every $s-p \leq i \leq \min (s-p+l-1, s)$. Moreover, $\left(F^{[s]}(t, X)\right)_{t \in G}$ is $\infty$-extensible if and only if there are constants $d_{i} \in \mathbb{K}$ for $s-p \leq i \leq s)$ such that $a_{i}=d_{i} c_{p+2}$ for every $s-p \leq i \leq s$.

Remark 1. Note that Corollary 2 states that in the case $c_{1}=1$ a one-parameter group of truncated formal power series $F^{[s]}(t, X)=$ $\sum_{i=1}^{s} c_{i}(t) X^{i}$ is $\infty$-extensible if and only if the coefficient functions $c_{n}$ for $n \in|s-p, s|$ depend only (cf. Theorem 1(b)) on $c_{p+2}$ and on some constants, but not on another additive function $a_{n}$.

We will finish this section with the following very useful and very simple result, which we are able to derive from Corollary 2.

Corollary 3. Let $F^{[s]}(t, X)=\sum_{i=1}^{s} c_{i}(t) X^{i}$ for $t \in G$, where $c_{1}: G \rightarrow$ $\mathbb{K} \backslash\{0\}, c_{i}: G \rightarrow \mathbb{K}$ for $2 \leq i \leq s$, be a one-parameter group of $s$-truncated formal power series. Then the truncated one-parameter group

$$
\left(\left(F^{[s]}\right)^{\left[\frac{1}{2} s\right]+1}(t, X)\right)_{t \in G}
$$

is $\infty$-extensible.

Proof. Assume that $F^{[s]}(t, X)=\sum_{i=1}^{s} c_{i}(t) X^{i}$ is a one-parameter group of $s$-truncated formal power series. The case $c_{1} \neq 1$ is trivial. Assume that $c_{1}=1$. Then (see Theorem 1(b)) the coefficient functions $c_{n}$ with $n \in\left|p+2,\left[\frac{1}{2} s\right]+1\right|$ depend only on $c_{p+2}$, but not on another additive function $a_{n}$.

\section{Stability of the Translation Equation in $\mathbb{K} \llbracket X \rrbracket$}

Now we prove results stating Hyers-Ulam stability of the translation equation in a ring of formal power series. We consider a definition of stability of functional equations, which is more general than $r$-stability.

Definition 7. The translation equation (1) in a ring of formal power series is called stable if for every positive integer $N$ we find a positive integer $M$ such that for every family $F(t, X)=\sum_{i=1}^{\infty} c_{i}(t) X^{i}, t \in G$ of formal power series such that

$$
\operatorname{ord}\left(F\left(t_{1}+t_{2}, X\right)-F\left(t_{1}, F\left(t_{2}, X\right)\right)\right)>M \quad \text { for every } \quad t_{1}, t_{2} \in G,
$$


there is a one-parameter group of formal power series $\bar{F}(t, X)=$ $\sum_{i=1}^{\infty} \bar{c}_{i}(t) X^{i}$ such that

$$
\operatorname{ord}(F(t, X)-\bar{F}(t, X))>N \quad \text { for } \quad t \in G
$$

or, equivalently, $c_{i}=\bar{c}_{i}$ for $1 \leq i \leq N$.

We will assume that $(G,+)$ is an abelian group satisfying (A). First let us assume additionally that $\operatorname{dim}_{\mathbb{K}} \mathcal{A}(G, \mathbb{K}) \leq 1$. Then, on account of Corollary 1, we have

Theorem 2. Assume that $(G,+)$ is an abelian group satisfying (A) and such that $\operatorname{dim}_{\mathbb{K}} \mathcal{A}(G, \mathbb{K}) \leq 1$. Let $M \in \mathbb{N}$ be fixed. If $(F(t, x))_{t \in G}$ is a family of formal power series such that

$$
\operatorname{ord}\left(F\left(t_{1}+t_{2}, X\right)-F\left(t_{1}, F\left(t_{2}, X\right)\right)\right)>M \quad \text { for every } t_{1}, t_{2} \in G,
$$

then there exists a one-parameter group of formal power series $(\bar{F}(t, X))_{t \in G}$ such that

$$
\operatorname{ord}(F(t, X)-\bar{F}(t, X))>M \quad \text { for } \quad t \in G \text {. }
$$

Proof. Note that the condition (3) means that for a family $(F(t, X))_{t \in G}$ with $F(t, X)=\sum_{i=1}^{\infty} c_{i}(t) X^{i}$, its $M$-truncation $\left(F^{[M]}(t, X)\right)_{t \in G}$ satisfies (2) with $s=M$. Then on account of Corollary 1 we know that $(F(t, X))_{t \in G}$ is $\infty$-extensible. This means that we find a one-parameter group of formal power series $(\bar{F}(t, X))_{t \in G}$ satisfying (4).

If we do not assume that $\operatorname{dim}_{\mathbb{K}} \mathcal{A}(G, \mathbb{K}) \leq 1$, we have

Theorem 3. Assume that $(G,+)$ is an abelian group satisfying (A). Let $M \in \mathbb{N}$ be fixed. If $(F(t, x))_{t \in G}$ is a family of formal power series satisfying (3), then there exists a one-parameter group of formal power series $(\bar{F}(t, X))_{t \in G}$ such that

$$
\operatorname{ord}(F(t, X)-\bar{F}(t, X))>\left[\frac{1}{2} M\right]+1 \quad \text { for } \quad t \in G .
$$

Proof. As in the proof of Theorem 2, the condition (3) means that for a family $(F(t, X))_{t \in G}$ with $F(t, X)=\sum_{i=1}^{\infty} c_{i}(t) X^{i}$, its $M$-truncation $\left(F^{[M]}(t, X)\right)_{t \in G}$ satisfies (2) with $s=M$. Then on account of Corollary 3 we know that the truncation

$$
\left(\left(F^{[M]}\right)^{\left[\frac{1}{2} M\right]+1}(t, X)\right)_{t \in G}
$$

is $\infty$-extensible. This means that we find a one-parameter group of formal power series $(\bar{F}(t, X))_{t \in G}$ such that (5) holds. 
Remark 2. Let us note that the one-parameter group of formal power series $(\bar{F}(t, X))_{t \in G}$ satisfying (4) and (5), respectively, which exists by Theorems 2 and 3 is not unique (cf. Theorem 1). This follows from the fact that constants $h_{n}$ and $\lambda_{n}$, respectively, can be chosen arbitrarily for $n>M$.

Remark 3. The estimations (4) and (5) obtained in the Theorems 2 and 3 are the best possible. First let us consider the case when $\operatorname{dim}_{\mathbb{K}} \mathcal{A}(G, \mathbb{K}) \leq 1$. Fix $M \in \mathbb{N}$ arbitrarily. Let $c_{1}=1, c_{i}=0$ for $i \in|2, M|, c_{M+1}: G \rightarrow \mathbb{K}$ let be an arbitrary function which is not additive and take arbitrary functions $c_{i}: G \rightarrow \mathbb{K}$ for $i \in|M+2, \infty|$. Put $F(t, X)=\sum_{i=1}^{\infty} c_{i}(t) X^{i}$. It is clear (cf. Theorem 1) that

$$
\operatorname{ord}\left(F\left(t_{1}+t_{2}, X\right)-F\left(t_{1}, F\left(t_{2}, X\right)\right)\right)=M+1 \quad \text { for } \quad t_{1}, t_{2} \in G \text {. }
$$

Let $\bar{c}_{1}=1$ and $\bar{c}_{i}=0$ for every $i \in|2, \infty|$. Then $\bar{F}(t, X)=$ $\sum_{i=1}^{\infty} \bar{c}_{i}(t) X^{i}$ is a one-parameter group of formal power series such that

$$
\operatorname{ord}(F(t, X)-\bar{F}(t, X))=M+1 \quad \text { for } \quad t \in G .
$$

Now assume that $\operatorname{dim}_{\mathbb{S}} \mathcal{A}(G, \mathbb{K}) \geq 2$. Fix $M \in \mathbb{N}$ arbitrarily. In the cases $M \in\{1,2\}$ works the same example as above. So assume that $M \geq 3$ and put $p:=[M / 2]-1$. Let $c_{1}=1, c_{i}=0$ for $i \in|2, p+1|$ and let $c_{p+2}: G \rightarrow \mathbb{K}$ be a nonzero additive function. Furthermore, let

$$
\begin{aligned}
c_{i}(t)= & h_{i} c_{p+2}(t)+L_{i}\left(c_{p+2}(t), h_{p+3}, \ldots, h_{i-p-1}\right), \\
& i \in|p+3, M-p-1|, \\
c_{i}(t)= & a_{i}(t)+L_{i}\left(c_{p+2}(t), h_{p+3}, \ldots, h_{i-p-1}\right), \quad i \in|M-p, M|,
\end{aligned}
$$

where $\left(L_{n}^{p+2}\right)_{n \geq p+2}$ are the polynomials from Theorem 1, $\left(h_{n}\right)_{n \in|p+3, M-p-1|}, h_{n} \in \mathbb{K}$ for $n \in|p+3, M-p-1|$, is a fixed sequence of constants and $a_{n}: G \rightarrow \mathbb{K}$ for $n \in|s-p, s|$ are fixed additive functions such that $a_{M-p} \neq b c_{p+2}$ for any $b \in \mathbb{K}$ (this is possible because of the assumption $\operatorname{dim}_{\mathbb{K}} \mathcal{A}(G, \mathbb{K}) \geq 2$ ). Finally, let $c_{i}$ for $i \geq M+1$ be arbitrary. Put $F(t, X)=\sum_{i=1}^{\infty} c_{i}(t) X^{i}$. It is clear (cf. Theorem 1) that

$$
\operatorname{ord}\left(F\left(t_{1}+t_{2}, X\right)-F\left(t_{1}, F\left(t_{2}, X\right)\right)\right)=M+1 \quad \text { for } \quad t_{1}, t_{2} \in G,
$$

because $a_{M-p} \neq b c_{p+2}$ for any $b \in \mathbb{K}$. Let $\bar{c}_{i}=c_{i}$ for every $i \in|1, M-p-1|$ and put

$$
\begin{gathered}
\bar{c}_{i}(t)=h_{i} c_{p+2}(t)+L_{i}\left(c_{p+2}(t), h_{p+3}, \ldots, h_{i-p-1}\right) \\
\text { for } i \in|M-p-1, \infty| .
\end{gathered}
$$


Then $\bar{F}(t, X)=\sum_{i=1}^{\infty} \bar{c}_{i}(t) X^{i}$ is a one-parameter group of formal power series such that

$$
\operatorname{ord}(F(t, X)-\bar{F}(t, X))=M-p=\left[\frac{M}{2}\right]+1 \quad \text { for } \quad t \in G .
$$

\section{References}

[1] FORTI, G. L. (1995) Hyers-Ulam stability of functional equations in several variables. Aequationes Math. 50: 143-190

[2] HyERS, D. H., ISAC, G., RASIAS, TH. M. (1998) Stability of Functional Equations in Several Variables (Progress in Nonlinear Differential Equations and Their Applications, Vol. 34). Birkhäuser, Boston Basel Berlin

[3] JABŁOŃSKI, W. (1999) On extensibility of some homomorphisms. Rocznik Nauk.-Dydak. WSP w Krakowie 16/207: 35-43

[4] JABŁOŃSKI, W., REICH, L. (2005) On the solutions of the translation equation in rings of formal power series. Abh. Math. Sem. Univ. Hamburg 75: 179-201

[5] JABŁOŃSKI, W., REICH, L. (2005) On the form of homomorphisms into the differential group $L_{s}^{1}$ and their extensibility. Result. Math. 47: 61-68

[6] JABŁOŃSKI, W., REICH, L.: On homomorphisms of an Abelian group into the group of invertible formal power series (manuscript)

[7] ReICH, L. (1991) Problem. Aequationes Math. 41: 248-310

[8] Ulam, S. M. (1960) A Collection of Mathematical Problems. Interscience, New York London

Authors' addresses: Dr. Wojciech Jabłoński, Department of Mathematics, University of Rzeszów, Rejtana 16 A, 35-310 Rzeszów, Poland. E-Mail: wojciech@univ. rzeszow.pl; Prof. Dr. Ludwig Reich, Institute of Mathematics, Karl-FranzensUniversity Graz, Heinrichstrasse 36, 8010 Graz, Austria. E-Mail: ludwig.reich@ uni-graz.at. 